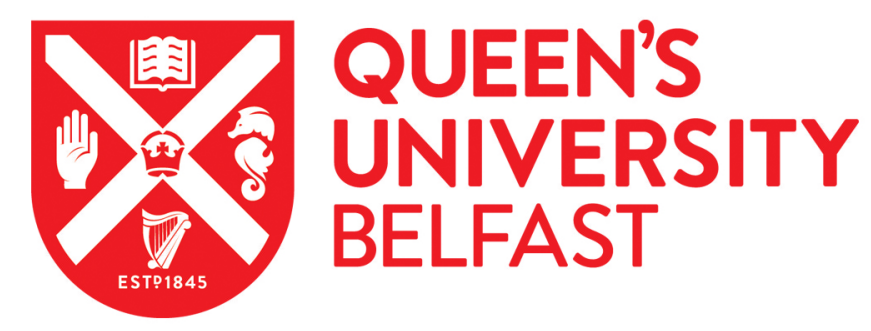

\title{
Observation of Ultrafast Charge Migration in an Amino Acid
}

Belshaw, L., Calegari, F., Duffy, M., Trabattoni, A., Poletto, L., Nisoli, M., \& Greenwood, J. (2012). Observation of Ultrafast Charge Migration in an Amino Acid. Journal of Physical Chemistry Letters, 3(24), 3751-3754. https://doi.org/10.1021/jz3016028

Published in:

Journal of Physical Chemistry Letters

Document Version:

Publisher's PDF, also known as Version of record

Queen's University Belfast - Research Portal:

Link to publication record in Queen's University Belfast Research Portal

\section{General rights}

Copyright for the publications made accessible via the Queen's University Belfast Research Portal is retained by the author(s) and / or other copyright owners and it is a condition of accessing these publications that users recognise and abide by the legal requirements associated with these rights.

Take down policy

The Research Portal is Queen's institutional repository that provides access to Queen's research output. Every effort has been made to ensure that content in the Research Portal does not infringe any person's rights, or applicable UK laws. If you discover content in the Research Portal that you believe breaches copyright or violates any law, please contact openaccess@qub.ac.uk. 


\title{
Observation of Ultrafast Charge Migration in an Amino Acid
}

\author{
Louise Belshaw, ${ }^{\dagger, \|}$ Francesca Calegari, ${ }^{\ddagger}, \|$ Martin J. Duffy, ${ }^{\dagger, \|}$ Andrea Trabattoni, ${ }^{\ddagger}$ Luca Poletto, ${ }^{\S}$ \\ Mauro Nisoli, ${ }^{\ddagger}$ and Jason B. Greenwood ${ }^{*}{ }^{\dagger}$ \\ ${ }^{\dagger}$ Centre for Plasma Physics, School of Maths and Physics, Queen’s University Belfast, BT7 1NN, United Kingdom \\ ${ }^{\ddagger}$ Politecnico di Milano, Department of Physics, Institute of Photonics and Nanotechnologies, CNR-IFN, I-20133 Milan, Italy \\ ${ }^{\S}$ Institute of Photonics and Nanotechnologies, CNR-IFN, I-35131 Padua, Italy
}

\section{Supporting Information}

\begin{abstract}
We present the first direct measurement of ultrafast charge migration in a biomolecular building block - the amino acid phenylalanine. Using an extreme ultraviolet pulse of $1.5 \mathrm{fs}$ duration to ionize molecules isolated in the gas phase, the location of the resulting hole was probed by a 6 fs visible/near-infrared pulse. By measuring the yield of a doubly charged ion as a function of the delay between the two pulses, the positive hole was observed to migrate to one end of the cation within $30 \mathrm{fs}$. This process is likely to originate from even faster coherent charge oscillations in the molecule being dephased by bond stretching which eventually localizes the final position of the charge. This demonstration offers a clear template for observing and controlling this phenomenon in the future.
\end{abstract}

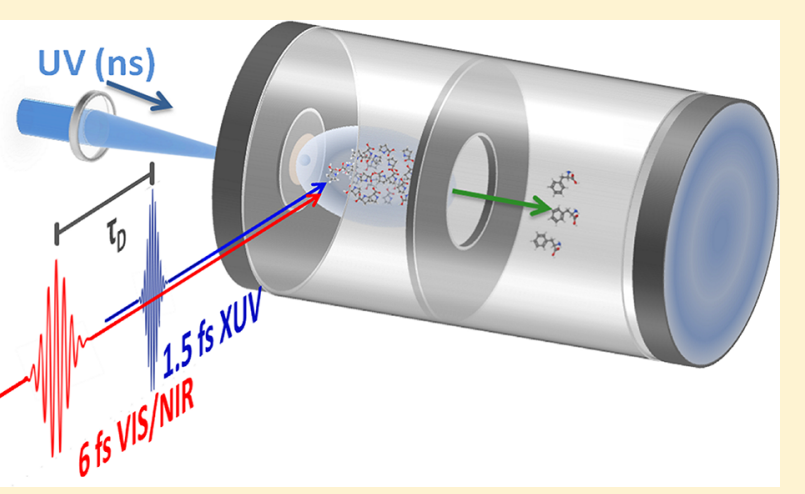

SECTION: Spectroscopy, Photochemistry, and Excited States

$\mathrm{T}$ ransfer of electronic charge within a single molecule is the fundamental initiator of many biological processes and chemical reactions. It plays a ubiquitous role in catalysis, ${ }^{1}$ DNA damage by ionizing radiation, ${ }^{2}$ photosynthesis, ${ }^{3}$ respiration, ${ }^{4}$ and photovoltaics ${ }^{5}$ and for switches based on molecular nanojunctions. ${ }^{6}$ How this process depends on the time scales, energetics, and molecular distances has been the subject of considerable research effort. ${ }^{7-9}$ The ability of molecules such as peptides and DNA to act as charge conduits is an intrinsic part of many biological processes. Charge can be transferred between two distant centers using covalently bonded molecules as a bridge. Given that these molecules are normally regarded as insulators, this can be a surprisingly efficient process and has led to considerable discussion of potential mechanisms such as superexchange and charge hopping. ${ }^{7,9}$

The study of intramolecular charge transfer in isolated, complex molecules was pioneered in the 1990s by Weinkauf and coworkers. They showed that if an electron is selectively ionized from a chromophore at the $\mathrm{C}$ terminal end of a peptide, then the location of the charge could be probed using the shift in absorption of the chromophore once charged. ${ }^{14,15}$ With this scheme, they were able to observe passage of charge through up to 12 sigma bonds in a quadrapeptide, but the temporal resolution was limited by the nanosecond laser pulse lengths. In a later experiment using pulse lengths of 200 and $120 \mathrm{fs}$ for the pump and probe, respectively, they were able to extract a 80 fs lifetime for downhill charge transfer from an ionized chromophore to the amine group in 2-phenylethyl- $N, N$ dimethylamine (PENNA). They suggested this was most likely due to transfer between electronic states through a conical intersection, ${ }^{16}$ which is reached by the nuclear wavepacket launched from the initial ionization.

The potential importance of even faster electron-transport mechanisms mediated by electronic wavepackets that can cross multiple molecular bonds has been recognized in a number of ground-breaking theoretical papers. ${ }^{10-13}$ If an electron is suddenly removed from an orbital of the neutral, then the molecule will be in a superposition of electronic states of the radical cation. The evolution of this electronic wavepacket that produces charge oscillations has been labeled charge migration to distinguish it from charge transfer mediated by nuclear motion. Depending on the cationic states contributing to the wavepacket and the conformation of the neutral molecule, charge migration across the full extent of the molecule has been predicted to take $5 \mathrm{fs}$ or less for a number of different molecules. ${ }^{10-13}$ This long-range coherent process is believed to be due to coupling of the single hole states to excited states that have large spatial extent (two hole, one particle configurations) due to strong electron correlation. ${ }^{13}$ Because these couplings are influenced by the geometry of the molecule, different initial conformations produce variations in the spatial and temporal behavior of the charge migration.

The recent development of few cycle ( $<7 \mathrm{fs}$ ) infrared laser pulses and extreme ultraviolet (XUV) attosecond pulses has

Received: October 7, 2012

Accepted: December 3, 2012

Published: December 3, 2012 
opened up the possibility of direct time domain measurements of electronic motion. This has been demonstrated for electron tunneling in atoms, photoelectron emission from surfaces, and charge oscillations in simple molecules; ${ }^{17-19}$ however, to date, there have been no experimental measurements of charge migration in complex molecules due to the difficulty of efficiently producing these labile species and the demanding temporal and spectral light pulse specifications required. In our experimental setup, we have overcome these challenges by combining a new attosecond laser beamline with a laserinduced acoustic desorption (LIAD) technique that is effective at producing clean plumes of isolated, neutral molecules for a range of biomolecular building blocks. ${ }^{20-22}$ In the present work, phenylalanine molecules were irradiated by a $1.5 \mathrm{fs} \mathrm{XUV}$ pump pulse with a photon energy in the range $16-40 \mathrm{eV}$, followed at a controllable delay time by a $6 \mathrm{fs}$ visible/nearinfrared (500-950 nm, VIS/NIR) probe pulse with an intensity of $8 \times 10^{12} \mathrm{~W} \mathrm{~cm}^{-2}$. The parent and fragment ions produced were then extracted into a linear time-of-flight device for mass analysis. Further experimental details can be found in the Supporting Information.

We chose phenylalanine as a model molecule for charge migration because its radical cation has two charge-acceptor sites with approximately the same binding energy located on the phenyl and amine groups, ${ }^{23}$ separated by two singly bonded carbons (Figure 1). Figure 2 shows the mass spectra obtained

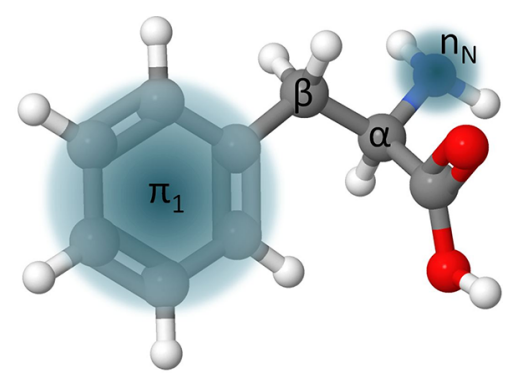

Figure 1. Three-dimensional structure of phenylalanine. The locations of the two highest occupied molecular orbitals are shown. These correspond to the $n_{\mathrm{N}}$ lone electron pair on the nitrogen of the amine group and $\pi_{1}$ of the phenyl group, separated by a bridge of two carbon atoms $(\alpha, \beta)$.

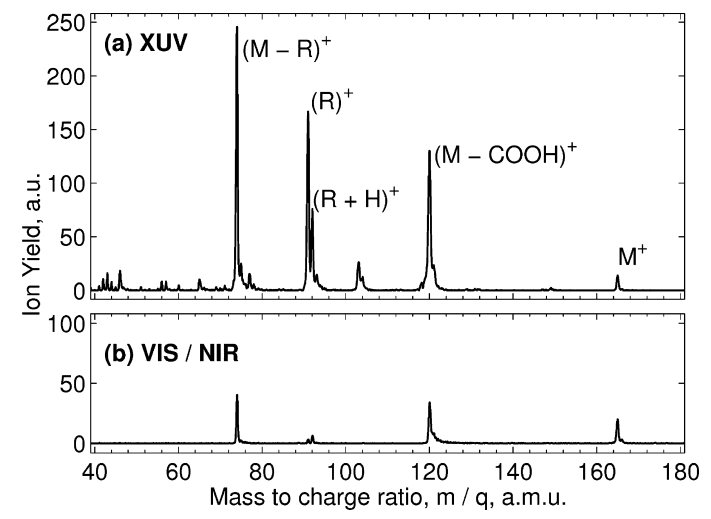

Figure 2. Mass spectra from ionization of phenylalanine. (a) XUV pulse only and (b) VIS/NIR pulse only. $M$ is the parent ion, with major fragments corresponding to loss of the carboxyl group (immonium ion $\mathrm{M}-\mathrm{COOH}$ ) and cleavage of the $\mathrm{C}_{\alpha}-\mathrm{C}_{\beta}$ bond (sidechain group $\mathrm{R}$ and $\mathrm{M}-\mathrm{R})$. individually from the XUV (a) and VIS/NIR (b) pulses. The main contributions correspond to the parent ion $\mathrm{M}^{+}(165 \mathrm{Da})$, loss of the carboxyl group yielding the immonium ion (M$\mathrm{COOH}=120)$, and breakage of the $\mathrm{C}_{\alpha}-\mathrm{C}_{\beta}$ bond with the charge residing on the amine $(\mathrm{M}-\mathrm{R}=74)$ or phenyl groups ( $\mathrm{R}$ $=91, \mathrm{R}+\mathrm{H}=92)$. The XUV pulse is capable of ionizing all valence and some inner shell orbitals, resulting in a wide range of fragment ions, as seen in Figure 2a. We particularly note peaks at $103\left(\mathrm{M}-\mathrm{COOH}, \mathrm{NH}_{3}\right), 77\left(\mathrm{C}_{6} \mathrm{H}_{5}^{+}\right)$, and $65\left(\mathrm{C}_{5} \mathrm{H}_{5}^{+}\right)$, which correspond to charge localized on the phenyl ring, and a small peak at 60 , which is due to the doubly charged immonium ion. To probe localization of the charge at the phenyl or amine sites, we have exploited differences in the excitation and ionization rates of these groups using the VIS/NIR pulse, as explained in the following paragraphs.

Figure 3 shows the yield of a number of different fragment ions relative to the largest peak in the spectrum at mass 74 .

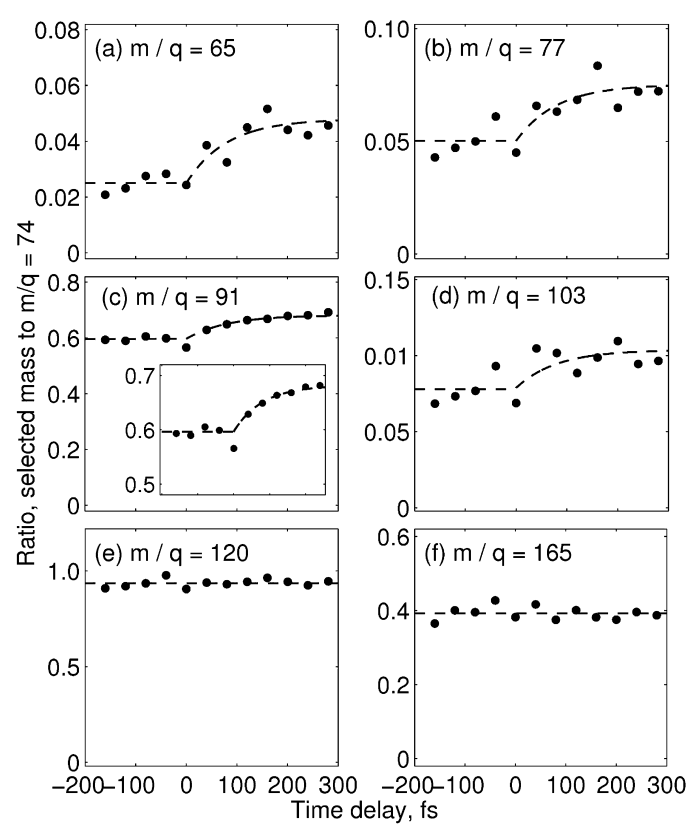

Figure 3. Yields of ions relative to the dominant ion in the spectrum (M-R) as a function of pump (XUV)-probe (VIS/NIR) delay. (a) Mass 65 - phenyl ring with $1 \mathrm{C}$ eliminated; (b) 77 - phenyl ring; (c) 91 - side-chain ions $\mathrm{R}$; (d) 103 - $\mathrm{M}-\mathrm{COOH}, \mathrm{NH}_{3}$; (e) 120 immonium ions $\mathrm{M}-\mathrm{COOH}$; and (f) 165 - parent ion M. Dotted lines in panels $\mathrm{a}-\mathrm{d}$ are simple exponential fits to the data with a time constant of $80 \mathrm{fs}$. The inset in panel c provides a magnified view for data close to time zero.

Whereas there is no temporal dependence in the parent (165) and immonium (120) ions, fragments corresponding to charge residing on the phenyl group $(65,77,91,103)$ increase gradually for positive delays. Within statistical uncertainties, the time constants for each of these fragments are consistent and give an overall weighted average of $80 \pm 20$ fs. This temporal evolution can be attributed to an internal conversion process into the $\pi_{1}$ state of the phenyl radical cation following initial ionization of a different orbital by the XUV pulse. It is known that the $\pi_{1}$ state of the phenyl radical cation absorbs strongly in the visible while the neutral phenyl and neutral and charged amine groups do not. ${ }^{15}$ Therefore, increasing the population of this state opens up the absorption by the broadband VIS/NIR pulse through resonant 1 photon transitions, thus enhancing 
the production of fragments corresponding to charge on the ring.

Figure 4 shows that the doubly charged immonium ion (mass 60) has a very sharp rise at $t=0$, followed by an

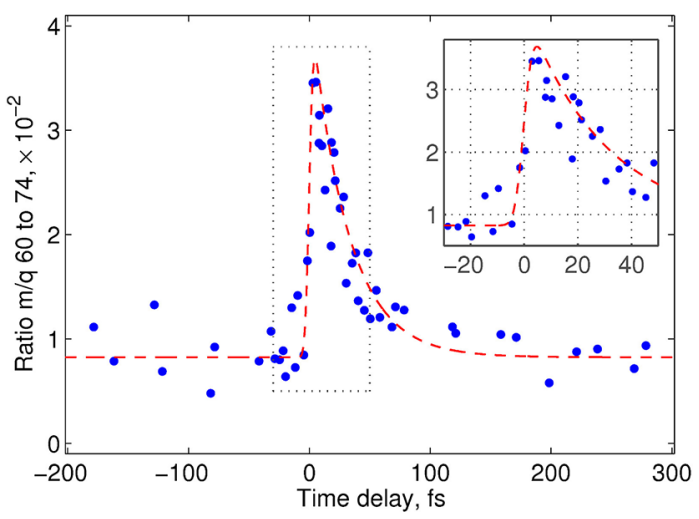

Figure 4. Yield of doubly charged immonium ion (mass 60) relative to the dominant ion in the spectrum (M-R, mass 74) as a function of pump (XUV)-probe (VIS/NIR) delay. The dotted line is a fit using a simple exponential decay convolved with a Gaussian with a width corresponding to the cross correlation of the two pulses $(\sim 6.2 \mathrm{fs})$. The time constant of the fit is $30 \mathrm{fs}$. The inset shows an expanded view of the points close to zero delay.

exponential decay with a short time constant of $30 \pm 5 \mathrm{fs}$. The identification of this peak as a doubly charged ion is confirmed by the peaks at 60.5 (an isotopologue) and 59.5 ( $\mathrm{H}$ loss), which have similar temporal behavior. There is no indication in the literature that double ionization of neutral amino acids can lead to a stable doubly charged parent ion. However, it is evident that loss of neutral $\mathrm{COOH}$ helps stabilize the doubly charged immonium ion, allowing it to have holes at both the amine and phenyl sites. Carboxyl loss is common following ionization of amino acids and is mediated by an $\alpha$-cleavage mechanism from charge residing on the amine group. ${ }^{24}$ In contrast with the XUV pump pulse, the VIS/NIR probe pulse is only capable of ionizing the lowest-lying orbitals (phenyl $\pi_{1}$, amine $\mathrm{n}_{\mathrm{N}}$ ) due to its moderate intensity. The delocalized nature of the $\pi_{1}$ phenyl orbital leads to a reduced rate of tunnel ionization due to the large dipole moment induced in the ring by the strong laser field. In organic molecules, this behavior manifests itself as higher saturation intensities for single ionization than would be expected for atoms with similar ionization potentials. ${ }^{25}$ Therefore, it is more probable that the atomic-like $n_{N}$ orbital is ionized by the VIS/NIR pulse than the $\pi_{1}$ orbital. $^{26}$

The yield of immonium dications is a particularly sensitive probe of charge location because the local ionization potential will increase as the hole approaches the amine group causing ionization by the VIS/NIR pulse to be suppressed. It is worth noting that fluctuations in the data close to time zero visible in the inset of Figure 4 might be produced by charge oscillations to and from the amine site with a period close to the duration of the probe pulse ( $\sim 6 \mathrm{fs})$, although this needs to be confirmed by additional experiments with improved statistics. Although such electronic motion is faster than nuclear rearrangement, the electron wavepacket would be expected to lose coherence as bonds are stretched, resulting in the charge finally settling at the amine site. We propose that the measured $30 \mathrm{fs}$ time constant is therefore a consequence of the sensitivity of charge migration to the nuclear dynamics. This is consistent with the model of charge migration in PENNA described by Lünnmeman et al., ${ }^{12}$ who showed that charge oscillations between the phenyl and amine sites are strongly influenced by the $\mathrm{C}_{\alpha}-\mathrm{C}_{\beta}$ bond length.

We have identified two separate ultrafast processes in isolated phenylalanine molecules. Following ionization of phenylalanine with an XUV photon, transfer into the $\pi_{1}$ state of the phenyl group in $\sim 80$ fs was measured through enhanced absorption of the VIS/NIR probe pulse, which we attribute to an internal conversion processes from higher lying states in the cation. In contrast, because the probe pulse preferentially ionizes the $n_{N}$ orbital, we have demonstrated that the double ionization yield is a very sensitive test for proximity of charge to the amine group. This was demonstrated by a dramatic increase in production of doubly charged immonium ions when the XUV pulse just precedes the VIS/NIR pulse, which reduces for longer delays with a time constant of $30 \mathrm{fs}$ due to migration of charge toward the amine group. Such a fast process is consistent with the model of ultrasfast coherent charge oscillations to and from the amine site being terminated by nuclear rearrangement. The use of attosecond pump pulses and few-optical cycle VIS/NIR probe pulses together with the implementation of the double ionization technique provides a powerful scheme capable of studying charge migration, which will allow this phenomenon, and its consequences for a range of biological processes, to be more fully understood. In the future such dynamics could be coherently manipulated with additional ultrashort pulses so that the final destination of the charge could be steered, giving unprecedented quantum control over any subsequent chemical reactivity.

\section{ASSOCIATED CONTENT}

\section{Supporting Information}

Supplementary figures and legends, supplementary methods, supplementary discussion, and supplementary references. This material is available free of charge via the Internet at http:// pubs.acs.org.

\section{AUTHOR INFORMATION}

\section{Corresponding Author}

*E-mail: j.greenwood@qub.ac.uk.

\section{Author Contributions}

"These authors contributed equally to this work.

Notes

The authors declare no competing financial interests.

\section{ACKNOWLEDGMENTS}

We acknowledge the support from LASERLAB-EUROPE (grant agreement $\mathrm{n}^{\circ}$ 284464, EC's Seventh Framework Programme), the European Research Council under the European Community's Seventh Framework Programme (FP7/2007-2013)/ERC grant agreement no. 227355 ELYCHE, the Leverhulme Trust, and the Department of Employment and Learning Northern Ireland.

\section{REFERENCES}

(1) Bauer, A.; Westkämper, F.; Grimme, S.; Bach, T. Catalytic Enantioselective Reactions Driven by Photoinduced Electron Transfer. Nature 2005, 436, 1139-1140.

(2) Becker, D.; Adhikary, A.; Sevilla, M. D. The Role of Charge and Spin Migration in DNA Radiation Damage. Charge Migration in DNA; Chakraborty, T., Ed.; Springer: New York, 2007; pp 139-175.

(3) Eberhard, S.; Finazzi, G.; Wollmann, F. A. The Dynamics of Photosynthesis. Annu. Rev. Genet. 2008, 42, 463-515. 
(4) Cordes, M.; Giese, B. Electron Transfer in Peptides and Proteins. Chem. Soc. Rev. 2009, 38, 892-901.

(5) Durrant, J. R.; Clarke, T. M. Charge Photogeneration in Organic Solar Cells. Chem. Rev. 2010, 110, 6736-6767.

(6) Kornyshev, A. A.; Kuznetsov, A. M.; Ulstrup, J. In Situ Superexchange Electron Transfer Through a Single Molecule: A Rectifying Effect. Proc. Natl. Acad. Sci. U.S.A. 2006, 103, 6799-6804.

(7) Marcus, R. A. Electron Transfer Reactions in Chemistry: Theory and Experiment (Nobel Lecture). Angew. Chem., Int. Ed. Engl. 1993, $32,1111-1121$.

(8) Bixner, O.; Lukes, V.; Mancal, T.; Hauer, J.; Milota, F.; Fischer, M.; Pugliesi, I.; Bradler, M.; Schmid, W.; Riedle, E.; et al. Ultrafast Photo-Induced Charge Transfer Unveiled by Two-Dimensional Electronic Spectroscopy. J. Chem. Phys. 2012, 136, 204503.

(9) Giese, B.; Graber, M.; Cordes, M. Electron Transfer in Peptides and Proteins. Curr. Opin. Chem. Biol. 2008, 12, 755-759.

(10) Remacle, F.; Levine, R. D. An Electronic Time Scale in Chemistry. Proc. Natl. Acad. Sci. U.S.A. 2006, 103, 6793-6798.

(11) Lünnemann, S.; Kuleff, A. I.; Cederbaum, L. S. Charge Migration Following Ionization in Systems with Chromophore-Donor and Amine-Acceptor Sites. J. Chem. Phys. 2008, 129, 104305.

(12) Lünnemann, S.; Kuleff, A. I.; Cederbaum, L. S. Ultrafast Charge Migration in 2-Phenylethyl-N,N-dimethylamine. Chem. Phys. Lett. 2008, 450, 232-235.

(13) Lünnemann, S.; Kuleff, A. I.; Cederbaum, L. S. ElectronCorrelation-Driven Charge Migration in Oligopeptides. Chem. Phys. 2012, DOI: http://dx.doi.org/10.1016/j.chemphys.2012.02.019.

(14) Weinkauf, R.; Aicher, P.; Wesley, G.; Grotemeyer, J.; Schlag, E. W. Femtosecond Versus Nanosecond Multiphoton Ionization and Dissociation of Large Molecules. J. Phys. Chem. 1994, 98, 8381-8391.

(15) Weinkauf, R.; et al. Highly Efficient Charge Transfer in Peptide Cations in the Gas Phase: Threshold Effects and Mechanism. J. Phys. Chem. 1996, 100, 18567-18585.

(16) Lehr, L.; Horneff, T.; Weinkauf, R.; Schlag, E. W. Femtosecond Dynamics after Ionization: 2-Phenylethyl-N,N-dimethylamine as a Model System for Nonresonant Downhill Charge Transfer in Peptides. J. Phys. Chem. A 2005, 109, 8074-8080.

(17) Krausz, F.; Ivanov, M. Attosecond Physics. Rev. Mod. Phys. 2009, 81, 163-234.

(18) Nisoli, M.; Sansone, G. New Frontiers in Attosecond Science. Progr. Quantum Elect. 2009, 33, 17-59.

(19) Kling, M. F.; Vrakking, M. J. J. Attosecond Electron Dynamics. Annu. Rev. Phys. Chem. 2008, 59, 463-492.

(20) Calvert, C. R.; Belshaw, L.; Duffy, M. J.; Kelly, O.; King, R. B.; Smyth, A. G.; Kelly, T. J.; Costello, J. T.; Timson, D. J.; Bryan, W. A.; et al. LIAD-fs Scheme for Studies of Ultrafast Laser Interactions with Gas Phase Biomolecules. Phys. Chem. Chem. Phys. 2012, 14, 62896297.

(21) Calvert, C. R.; Kelly, O.; Duffy, M. J.; Belshaw, L.; King, R. B.; Williams, I. D.; Greenwood, J. B. LIAD-fs: A Novel Method for Studies of Ultrafast Processes in Gas Phase Neutral Biomolecules. J. Phys.: Conf. Ser. 2012, 388, 012032.

(22) Belshaw, L.; Kelly, O.; Duffy, M. J.; King, R. B.; Kelly, T. J.; Costello, J. C.; Williams, I. D.; Calvert, C. R.; Greenwood, J. B. Ionisation and Fragmentation of Small Biomolecules with Femtosecond Laser Pulses. Springer Proc. Phys. 2013, 125, 309-312.

(23) Plekan, O.; Feyer, V.; Richter, R.; Coreno, M.; Prince, K. C. Valence Photoionization and Photofragmentation of Aromatic Amino Acids. Mol. Phys. 2008, 106, 1143-1153.

(24) Vorsa, V.; Kono, T.; Willey, K. F.; Winograd, N. Femtosecond Photoionization of Ion Beam Desorbed Aliphatic and Aromatic Amino Acids: Fragmentation via $\alpha$-Cleavage Reactions. J. Phys. Chem. B 1999, 103, 7889-7895.

(25) Hankin, S. M.; Villeneuve, D. M.; Corkum, P. B.; Rayner, D. M. Intense-Field Laser Ionization Rates in Atoms and Molecules. Phys. Rev. A 2001, 64, 013405.

(26) Mathur, D.; Hatamoto, T.; Okunishi, M.; Prumper, G.; Lischke, T.; Shimada, K.; Ueda, K. Ionization of Linear Alcohols by Strong Optical Fields. J. Phys. Chem. A 2007, 111, 9299-9306. 